\title{
Edmonds maps on the Fricke-Macbeath curve
}

\author{
Rubén A. Hidalgo * \\ Departamento de Matemática y Estadística, Universidad de La Frontera, \\ Casilla 54-D, 4780000 Teтисо, Chile
}

Received 4 June 2013, accepted 27 December 2014, published online 4 February 2015

\begin{abstract}
In 1985, L. D. James and G. A. Jones proved that the complete graph $K_{n}$ defines a clean dessin d'enfant (the bipartite graph is given by taking as the black vertices the vertices of $K_{n}$ and the white vertices as middle points of edges) if and only if $n=p^{e}$, where $p$ is a prime. Later, in 2010, G. A. Jones, M. Streit and J. Wolfart computed the minimal field of definition of them. The minimal genus $g>1$ of these types of clean dessins d'enfant is $g=7$, obtained for $p=2$ and $e=3$. In that case, there are exactly two such clean dessins d'enfant (previously known as Edmonds maps), both of them defining the Fricke-Macbeath curve (the only Hurwitz curve of genus 7) and both forming a chiral pair. The uniqueness of the Fricke-Macbeath curve asserts that it is definable over $\mathbb{Q}$, but both Edmonds maps cannot be defined over $\mathbb{Q}$; in fact they have as minimal field of definition the quadratic field $\mathbb{Q}(\sqrt{-7})$. It seems that no explicit models for the Edmonds maps over $\mathbb{Q}(\sqrt{-7})$ are written in the literature. In this paper we start with an explicit model $X$ for the FrickeMacbeath curve provided by Macbeath, which is defined over $\mathbb{Q}\left(e^{2 \pi i / 7}\right)$, and we construct an explicit birational isomorphism $L: X \rightarrow Z$, where $Z$ is defined over $\mathbb{Q}(\sqrt{-7})$, so that both Edmonds maps are also defined over that field.
\end{abstract}

Keywords: Riemann surface, algebraic curve, dessin d'enfant.

Math. Subj. Class.: 30F20, 30F10, 14Q05, 14H45, 14E05

\section{Introduction}

A dessin d'enfant $D$ on a closed orientable surface is given by a bipartite map on it (vertices will be colored black and white). The dessin d'enfant is called clean if the white vertices have all valence 2 .

A Belyi curve is an irreducible non-singular complex projective algebraic curve (i.e. a closed Riemann surface) $S$ admitting a non-constant meromorphic map $\beta: S \rightarrow \widehat{\mathbb{C}}$ with

* Partially supported by Project Fondecyt 1150003.

E-mail address: ruben.hidalgo@ufrontera.cl (Rubén A. Hidalgo) 
at most three branch values; which we assume to be inside the set $\{\infty, 0,1\}$; we say that $(S, \beta)$ is a Belyi pair. Two Belyi pairs $\left(S_{1}, \beta_{1}\right)$ and $\left(S_{2}, \beta_{2}\right)$ are called equivalent, denoted this by the symbol $\left(S_{1}, \beta_{1}\right) \cong\left(S_{2}, \beta_{2}\right)$, if there is an isomorphism $f: S_{1} \rightarrow S_{2}$ so that $\beta_{2} \circ f=\beta_{1}$.

A subfield $\mathbb{K}$ of $\overline{\mathbb{Q}}$ is called a field of definition of a Belyi pair $(S, \beta)$ if there an equivalent Belyi pair $(\widehat{S}, \widehat{\beta})$ where $\widehat{S}$ and $\widehat{\beta}$ are both defined over $\mathbb{K}$. As a consequence of Belyi's theorem [1], the field of algebraic numbers $\overline{\mathbb{Q}}$ is a field of definition of every Belyi pair.

Each Belyi pair $(S, \beta)$ defines a dessin d'enfant on $S$ by taking the edges as the components of $\beta^{-1}((0,1))$, the black vertices as the points in $\beta^{-1}(0)$ and the white vertices as the points in $\beta^{-1}(1)$. Conversely, as a consequence of the uniformization theorem, every dessin d'enfant on a closed orientable surface induces a (unique up to isomorphisms) Riemann surface structure (being a Belyi curve) and a Belyi map so that the original dessin d'enfant is homotopic to the one associated to the Belyi pair [11, 15].

A field of definition of a dessin d'enfant is a field of definition of the corresponding Belyi pair.

As there is a natural (faithful) action of the absolute Galois group $\operatorname{Gal}(\overline{\mathbb{Q}} / \mathbb{Q})$ on the collection of Belyi pairs [13], it also provides a (faithful) action on dessins d'enfant. This action may help in the study of the internal structure of the group $\operatorname{Gal}(\overline{\mathbb{Q}} / \mathbb{Q})$ in terms of combinatorial data.

Let us consider a dessin d'enfant $D$, which is defined by the Belyi pair $(S, \beta)$. By Belyi's theorem, we may assume that both $S$ and $\beta$ are defined over $\overline{\mathbb{Q}}$. The field of moduli of $D$ is then defined as the fixed field of the subgroup $\left\{\sigma \in \operatorname{Gal}(\overline{\mathbb{Q}} / \mathbb{Q}):(S, \beta) \cong\left(S^{\sigma}, \beta^{\sigma}\right)\right\}$. The field of moduli of $D$ is always contained in any field of definition of it, but it may be that the field of moduli is not a field of definition of it. Both, the computation of the field of moduli of a dessin d'enfant and to decide if the dessin d'enfant can be defined over it, are in general difficult problems. If the dessin d'enfant is regular, that is, the Belyi map $\beta$ is a Galois branched cover, then J. Wolfart [19] proved that $D$ can be defined over its field of moduli. Also, if the dessin d'enfant has no non-trivial automorphisms, then it is definable over its field of moduli as a consequence of Weil's descent theorem [16]. So, the problem to decide if the field of moduli is a field of definition appears when it has non-trivial automorphisms but it is non-regular.

In 1985, L. D. James and G. A. Jones [10] proved that the complete graph $K_{n}$ defines a clean dessin d'enfant (the bipartite graph is given by taking as the black vertices the vertices of $K_{n}$ and the white vertices as middle points of edges) if and only if $n=p^{e}$, where $p$ is a prime. Later, in 2010, G. A. Jones, M. Streit and J. Wolfart [12] computed the minimal field of definition of such clean dessins d'enfant. The minimal genus $g>1$ of these types of clean dessins d'enfants is $g=7$, obtained for $p=2$ and $e=3$. In that case, there are exactly two (non-equivalent) such dessins (previously known as Edmonds maps), both of them defining the Fricke-Macbeath curve (the only Hurwitz curve of genus 7) and both forming a chiral pair. The uniqueness of the Fricke-Macbeath curve asserts that it is definable over $\mathbb{Q}$, but each of the two Edmonds maps cannot be defined over $\mathbb{Q}$; they have as minimal field of definition the quadratic field $\mathbb{Q}(\sqrt{-7})$ [12]. No explicit models for the Edmonds maps over $\mathbb{Q}(\sqrt{-7})$ seems to be written in the literature.

In Section 2 we recall an explicit model $X$ for the Fricke-Macbeath curve provided by Macbeath, which is defined over $\mathbb{Q}\left(e^{2 \pi i / 7}\right)$, and describe both Edmonds maps. We also provide (as matter of interest for specialists) two different equations, over $\mathbb{Q}$, for the Fricke-Macbeath curve which were independently obtained by Bradley Brock (personal 
communication) and by Maxim Hendriks in his Ph.D. Thesis [7]. In Section 3 we provide an explicit birational isomorphism $L: X \rightarrow Z$, where $Z$ is defined over $\mathbb{Q}(\sqrt{-7})$. In this model we obtain that the two Belyi maps defining the two Edmonds maps are defined over $\mathbb{Q}$; in particular, this provides explicit models for both Edmonds maps over $\mathbb{Q}(\sqrt{-7})$ as desired. In Section 4 we provide an explicit birational isomorphism $\widehat{L}: X \rightarrow W$, where $W$ is defined over $\mathbb{Q}$. Unfortunately, the explicit equations over $\mathbb{Q}$ are not very simple (they are long ones) and they can be found in [9]. In Section 5 we construct a generalized Fermat curve $\widehat{S}$ of type $(2,6)$ [5] that covers the Fricke-Macbeath curve and we provide a description of the three elliptic curves appearing in the equations of $X$ given by Macbeath. Another model of the Fricke-Macbeath curve is also described.

\section{Macbeath's equations of the Fricke-Macbeath curve and the two Edmonds maps}

In this section we recall equations of the Fricke-Macbeath curve, obtained by Macbeath in [14], and we describe both Edmonds maps discovered in [12]. As a matter of interest to specialists, we also describe two different models over $\mathbb{Q}$, one obtained by Bradley Brock (personal communication) and the other by Maxim Hendriks in his Ph.D. Thesis [7].

\subsection{Hurwitz curves}

It is well known that $|\operatorname{Aut}(S)| \leq 84(g-1)$ (Hurwitz upper bound) if $S$ is a closed Riemann surface of genus $g \geq 2$. In the case that $|\operatorname{Aut}(S)|=84(g-1)$, one says that $S$ is a Hurwitz curve. In this last situation, the quotient orbifold $S / \operatorname{Aut}(S)$ has signature $(0 ; 2,3,7)$, that is, $S=\mathbb{H}^{2} / \Gamma$, where $\Gamma$ is a torsion free normal subgroup of finite index in the triangular Fuchsian group $\Delta=\left\langle x, y: x^{2}=y^{3}=(x y)^{7}=1\right\rangle$ acting as isometries of the hyperbolic plane $\mathbb{H}^{2}$.

Wiman [17] noticed that there is no Hurwitz curve in each genera $g \in\{2,4,5,6\}$ and there is exactly one Hurwitz curve (up to isomorphisms) of genus three, this being Klein's quartic $x^{3} y+y^{3} z+z^{3} x=0$; whose automorphisms group is the simple group $\operatorname{PSL}(2,7)$ (of order 168).

\subsection{Macbeath's equations of the Fricke-Macbeath curve}

In [14] Macbeath observed that in genus seven there is exactly one (up to isomorphisms) Hurwitz curve, called the Fricke-Macbeath curve; its automorphisms group is the simple group $\operatorname{PSL}(2,8)$, consisting of 504 symmetries. In the same paper, Macbeath computed the following explicit equations over $\mathbb{Q}(\rho)$, where $\rho=e^{2 \pi i / 7}$, for the Fricke-Macbeath curve (involving three particular elliptic curves):

$$
X=\left\{\begin{array}{l}
y_{1}^{2}=(x-1)\left(x-\rho^{3}\right)\left(x-\rho^{5}\right)\left(x-\rho^{6}\right) \\
y_{2}^{2}=\left(x-\rho^{2}\right)\left(x-\rho^{4}\right)\left(x-\rho^{5}\right)\left(x-\rho^{6}\right) \\
y_{4}^{2}=(x-\rho)\left(x-\rho^{3}\right)\left(x-\rho^{4}\right)\left(x-\rho^{5}\right)
\end{array}\right\} \subset \mathbb{C}^{4} .
$$

In Section 5 we provide a rough explanation about the elliptic curves in the above equations (different from the approach given in [14]) in geometric terms of the highest regular branched Abelian cover of the orbifold $X / G$ of signature $(0 ; 2,2,2,2,2,2,2)$. 
Another interesting fact on the Fricke-Macbeath curve is that its jacobian variety is isogenous to $E^{7}$ where $E$ is the elliptic curve with $j$-invariant $j(E)=1792$ ( $E$ does not have complex multiplication); see, for instance, [2]. There are not to many explicit examples of Riemann surfaces whose jacobian variety is isogenous to the product of elliptic curves (see [6]).

\subsection{Equations over $\mathbb{Q}$ of the Fricke-Macbeath curve}

The uniqueness up to isomorphisms of the Fricke-Macbeath curve asserts that its field of moduli is the field of rational numbers $\mathbb{Q}$. As quasiplatonic curves can be defined over their fields of moduli [19] and Hurwitz curve are quasiplatonic curves, it follows that the FrickeMacbeath curve can be defined over $\mathbb{Q}$. When the author put a first version of this paper in Arxiv [9] we didn't know of explicit equations of the Fricke-Macbeath curve over $\mathbb{Q}$. Later, Bradley Brock sent me an e-mail in which he told me that, using some suitable change of coordinates on the above equations for $X$, he was able to compute a plane equation for $X$ over $\mathbb{Q}$, with some simple nodes as singularities, given as

$$
1+7 x y+21 x^{2} y^{2}+35 x^{3} y^{3}+28 x^{4} y^{4}+2 x^{7}+2 y^{7}=0 .
$$

An automorphism of order 7 is given by $b(x, y)=\left(\rho x, \rho^{-1} y\right)$ and one of order two is given by $a_{1}(x, y)=(y, x)$.

The following model over $\mathbb{Q}$, for the Fricke-Macbeath curve, was recently computed by Maxim Hendriks in his Ph.D. Thesis [7]

$$
\left\{\begin{array}{c}
-x_{1} x_{2}+x_{1} x_{0}+x_{2} x_{6}+x_{3} x_{4}-x_{3} x_{5}-x_{3} x_{0}-x_{4} x_{6}-x_{5} x_{6}=0, \\
x_{1} x_{3}+x_{1} x_{6}-x_{2}^{2}+2 x_{2} x_{5}+x_{2} x_{0}-x_{3}^{2}+x_{4} x_{5}-x_{4} x_{0}-x_{5}^{2}=0, \\
x_{1}^{2}-x_{1} x_{3}+x_{2}^{2}-x_{2} x_{4}-x_{2} x_{5}-x_{2} x_{0}-x_{3}^{2}+x_{3} x_{6}+2 x_{5} x_{0}-x_{0}^{2}=0, \\
x_{1} x_{4}-2 x_{1} x_{5}+2 x_{1} x_{0}-x_{2} x_{6}-x_{3} x_{4}-x_{3} x_{5}+x_{5} x_{6}+x_{6} x_{0}=0, \\
x_{1}^{2}-2 x_{1} x_{3}-x_{2}^{2}-x_{2} x_{4}-x_{2} x_{5}+2 x_{2} x_{0}+x_{3}^{2}+x_{3} x_{6}+x_{4} x_{5}+x_{5}^{2}-x_{5} x_{0}-x_{6}^{2}=0, \\
-2 x_{1} x_{2}-x_{1} x_{0}-x_{5} x_{6} x_{5}+2 x_{1} x_{0}+2 x_{2} x_{3}-2 x_{3} x_{0}+2 x_{5} x_{6}-x_{6} x_{0}=0, \\
2 x_{1}^{2}+x_{1} x_{3}-x_{1} x_{6}+3 x_{2} x_{0}+x_{4} x_{5}-x_{4} x_{0}-x_{5}^{2}+x_{6}^{2}-x_{0}^{2}=0, \\
2 x_{1}^{2}-x_{1} x_{3}+x_{1} x_{6}+x_{2}^{2}+x_{2} x_{0}+x_{3}^{2}-2 x_{3} x_{6}+x_{4} x_{5}-x_{4} x_{0}+x_{5}^{2}-2 x_{5} x_{0}+x_{6}^{2}+x_{0}^{2}=0, \\
x_{1}^{2}+x_{1} x_{3}-x_{1} x_{6}+2 x_{2} x_{5}-3 x_{2} x_{0}+2 x_{3} x_{6}+x_{4}^{2}+x_{4} x_{5}-x_{4} x_{0}+x_{6}^{2}+3 x_{0}^{2}=0
\end{array}\right\} \subset \mathbb{P}^{6} .
$$

In Section 4 we provide an explicit birational isomorphism $\widehat{L}: X \rightarrow W$, where $W$ is defined over $\mathbb{Q}$. The explicit form of $\widehat{L}$ may be used to compute explicit equation for $W$; this can be done with MAGMA [3].

\subsection{A description of the two Edmonds maps}

In the above model $X$ of the Fricke-Macbeath curve it is easy to see a group $\mathbb{Z}_{2}^{3} \cong G=$ $\left\langle A_{1}, A_{2}, A_{3}\right\rangle<\operatorname{Aut}(X)$ where

$$
\begin{aligned}
& A_{1}\left(x, y_{1}, y_{2}, y_{4}\right)=\left(x,-y_{1}, y_{2}, y_{4}\right), \\
& A_{2}\left(x, y_{1}, y_{2}, y_{4}\right)=\left(x, y_{1},-y_{2}, y_{4}\right), \\
& A_{3}\left(x, y_{1}, y_{2}, y_{4}\right)=\left(x, y_{1}, y_{2},-y_{4}\right) .
\end{aligned}
$$

The quotient Riemann orbifold $X / G$ has signature $(0 ; 2,2,2,2,2,2,2)$, that is, is the Riemann sphere with exactly 7 cone points of order 2 . 
An automorphism of order 7 of the Fricke-Macbeath curve is given in such a model by

$$
B\left(x, y_{1}, y_{2}, y_{4}\right)=\left(\rho x, \rho^{2} y_{2}, \rho^{2} y_{4}, \rho^{2} \frac{y_{1} y_{2}}{\left(x-\rho^{5}\right)\left(x-\rho^{6}\right)}\right) .
$$

The automorphism $B$ normalizes $G$ and it induces, on the orbifold $X / G=\widehat{\mathbb{C}}$, the rotation $T(x)=\rho x$. Moreover, $X /\langle G, B\rangle$ has signature $(0 ; 2,7,7)$, that is, the group $\langle G, B\rangle$ defines a regular dessin d'enfant $(X, \beta)$, where $\beta\left(x, y_{1}, y_{2}, y_{4}\right)=x^{7}$ (this is one of the two Edmonds maps, but is defined over $\mathbb{Q}(\rho)$ ).

We may also see that $X$ admits the following anticonformal involution

$$
J\left(x, y_{1}, y_{2}, y_{4}\right)=\left(\frac{1}{\bar{x}}, \frac{\overline{y_{1}}}{\bar{x}^{2}}, \frac{\rho^{5} \overline{y_{2}}}{\bar{x}^{2}}, \frac{\rho^{3} \overline{y_{4}}}{\bar{x}^{2}}\right) .
$$

It can be seen that $J B J=B$ and $J A_{j} J=A_{j}$, for $j=1,2,3$. In this way, one gets another regular dessin d'enfant $(X, \delta)$, where $\delta\left(x, y_{1}, y_{2}, y_{4}\right)=1 / x^{7}$ (this is the other Edmonds map, again defined over $\mathbb{Q}(\rho))$.

As $\delta=C \circ \beta \circ J$, where $C(x)=\bar{x}$, we have that the two regular dessins d'enfant described above are chirals.

\section{An explicit model of the Edmonds maps over $\mathbb{Q}(\sqrt{-7})$}

In this section we will construct an explicit biregular isomorphism $L: X \rightarrow Z$, where $Z$ is defined over $\mathbb{Q}(\sqrt{-7})$, so that both Edmonds maps are defined over such a field.

Note that $\mathbb{Q}(\sqrt{-7})=\mathbb{Q}\left(\rho+\rho^{2}+\rho^{4}\right)$ since $\rho+\rho^{2}+\rho^{4}=\frac{1}{2}(\sqrt{-7}-1)$. Most of the computations have been carried out with MAGMA [3] or with MATHEMATICA [20].

\section{1}

Let $N=\operatorname{Gal}(\mathbb{Q}(\rho) / \mathbb{Q}(\sqrt{-7}))=\langle\tau\rangle \cong \mathbb{Z}_{3}$, where $\tau(\rho)=\rho^{2}$. If we set

$$
\vec{x}=\left(x_{1}, x_{2}, x_{3}, x_{4}\right)=\left(x, y_{1}, y_{2}, y_{4}\right),
$$

then it is not difficult to check that $\left\{f_{e}=I, f_{\tau}, f_{\tau^{2}}\right\}$ is a Weil datum (i.e., they satisfies the Weil co-cycle condition in Weil's descent theorem [16]) with respect to the Galois extension $\mathbb{Q}(\rho) / \mathbb{Q}(\sqrt{-7})$, where $I$ denotes the identity and

$$
\begin{aligned}
f_{\tau}(\vec{x}) & =\left(x, y_{1}, y_{4}, \frac{y_{2} y_{4}}{\left(x-\rho^{4}\right)\left(x-\rho^{5}\right)}\right), \\
f_{\tau^{2}}(\vec{x}) & =\left(x, y_{1}, \frac{y_{2} y_{4}}{\left(x-\rho^{4}\right)\left(x-\rho^{5}\right)}, y_{2}\right) .
\end{aligned}
$$

\section{2}

Let us consider the rational map

$$
\begin{aligned}
\Phi_{1}: X & \rightarrow \mathbb{C}^{12} \\
\left(x, y_{1}, y_{2}, y_{4}\right) & \mapsto(\vec{x}, \vec{w}, \vec{v}),
\end{aligned}
$$

where

$$
\vec{w}=\left(w_{1}, w_{2}, w_{3}, w_{4}\right)=f_{\tau}(\vec{x})
$$




$$
\vec{v}=\left(v_{1}, v_{2}, v_{3}, v_{4}\right)=f_{\tau^{2}}(\vec{x}) .
$$

We may see that $\Phi_{1}$ produces a birational isomorphism between $X$ and $\Phi_{1}(X)$ (its inverse is just the projection on the $\vec{x}$-coordinate). Equations defining the algebraic curve $\Phi_{1}(X)$ are the following ones

$$
\Phi_{1}(X)=\left\{\begin{array}{l}
x_{2}^{2}=\left(x_{1}-1\right)\left(x_{1}-\rho^{3}\right)\left(x_{1}-\rho^{5}\right)\left(x_{1}-\rho^{6}\right) \\
x_{3}^{2}=\left(x_{1}-\rho^{2}\right)\left(x_{1}-\rho^{4}\right)\left(x_{1}-\rho^{5}\right)\left(x_{1}-\rho^{6}\right) \\
x_{4}^{2}=\left(x_{1}-\rho\right)\left(x_{1}-\rho^{3}\right)\left(x_{1}-\rho^{4}\right)\left(x_{1}-\rho^{5}\right) \\
w_{1}=x_{1}, w_{2}=x_{2}, w_{3}=x_{4}, w_{4}=\frac{x_{3} x_{4}}{\left(x_{1}-\rho^{4}\right)\left(x_{1}-\rho^{5}\right)}, \\
v_{1}=x_{1}, v_{2}=x_{2}, v_{3}=\frac{x_{3} x_{4}}{\left(x_{1}-\rho^{4}\right)\left(x_{1}-\rho^{5}\right)}, v_{4}=x_{3}
\end{array}\right\}
$$

\section{3}

We consider the linear permutation action of $N$ on the coordinates of $\mathbb{C}^{12}$ defined by

$$
\Theta_{1}(\tau)(\vec{x}, \vec{w}, \vec{v})=(\vec{w}, \vec{v}, \vec{x}) .
$$

Let us notice that the stabilizer of $\Phi_{1}(X)$, with respect to the above permutation action, is trivial since

$$
\left\{\eta \in N: \Theta_{1}(\eta)\left(\Phi_{1}(X)\right)=\Phi_{1}(X)\right\}=\left\{\eta \in N: X^{\eta}=X\right\}=\{e\} .
$$

\section{4}

Each $\theta \in \operatorname{Gal}(\mathbb{C})$ induces a natural bijection

$$
\widehat{\theta}: \mathbb{C}^{12} \rightarrow \mathbb{C}^{12}:\left(y_{1}, \ldots, y_{12}\right) \mapsto\left(\theta\left(y_{1}\right), \ldots, \theta\left(y_{12}\right)\right) .
$$

It is not hard to see that $\widehat{\theta}(X)=X^{\theta}$.

\section{5}

If $\theta \in \operatorname{Gal}(\mathbb{C} / \mathbb{Q}(\sqrt{-7}))$, then we denote by $\theta_{N}$ is projection in $N$. With this notation, we see that the following diagram commutes (see also [8])

$$
\begin{array}{rll}
X & \stackrel{\Phi_{1}}{\rightarrow} & \Phi_{1}(X) \\
\downarrow f_{\theta_{N}} & & \downarrow \Theta_{1}\left(\theta_{N}\right) \\
X^{\theta_{N}} & \stackrel{\Phi_{1}^{\theta_{N}}}{\rightarrow} & \Theta_{1}\left(\theta_{N}\right)\left(\Phi_{1}(X)\right)=\Phi_{1}^{\theta_{N}}\left(X^{\theta_{N}}\right)=\Phi_{1}(X)^{\theta_{N}} \\
\downarrow \widehat{\theta}^{-1} & & \downarrow \widehat{\theta}^{-1} \\
X & \stackrel{\Phi_{1}}{\rightarrow} & \Phi_{1}(X)
\end{array}
$$

and, for every $\eta, \theta \in \operatorname{Gal}(\mathbb{C} / \mathbb{Q}(\sqrt{-7})))$, that

$$
(*) \quad \Theta_{1}\left(\eta_{N}\right) \circ \widehat{\theta}=\widehat{\theta} \circ \Theta_{1}\left(\eta_{N}\right) .
$$




\section{6}

A generating set of invariant polynomials for the linear action $\Theta_{1}(N)$ can be obtained with MAGMA as

$$
\begin{aligned}
& t_{1}=x_{1}+w_{1}+v_{1}, \quad t_{2}=x_{2}+w_{2}+v_{2} \\
& t_{3}=x_{3}+w_{3}+v_{3}, \quad t_{4}=x_{4}+w_{4}+v_{4} \\
& t_{5}=x_{1}^{2}+w_{1}^{2}+v_{1}^{2}, \quad t_{6}=x_{2}^{2}+w_{2}^{2}+v_{2}^{2} \\
& t_{7}=x_{3}^{2}+w_{3}^{2}+v_{3}^{2}, \quad t_{8}=x_{4}^{2}+w_{4}^{2}+v_{4}^{2} \\
& t_{9}=x_{1}^{3}+w_{1}^{3}+v_{1}^{3}, \quad t_{10}=x_{2}^{3}+w_{2}^{3}+v_{2}^{3} \\
& t_{11}=x_{3}^{3}+w_{3}^{3}+v_{3}^{3}, \quad t_{12}=x_{4}^{3}+w_{4}^{3}+v_{4}^{3}
\end{aligned}
$$

The map

$$
\begin{gathered}
\Psi_{1}: \mathbb{C}^{12} \rightarrow \mathbb{C}^{12} \\
(\vec{x}, \vec{w}, \vec{v}) \mapsto\left(t_{1}, \ldots, t_{12}\right)
\end{gathered}
$$

clearly satisfies the following properties:

$$
\left\{\begin{array}{l}
\Psi_{1}^{\tau^{j}}=\Psi_{1}, j=0,1,2 \\
\Psi_{1} \circ \Theta_{1}\left(\tau^{j}\right)=\Psi_{1}, j=0,1,2 .
\end{array}\right.
$$

Also (as we have chosen a set of generators of the invariant polynomials for the action of $\Theta_{1}(N)$ ), it holds that $\Psi_{1}$ is a branched regular cover with Galois group $N$. It turns out that, if we set $Z_{1}=\Psi_{1}\left(\Phi_{1}(X)\right)$ and $L_{1}=\Psi_{1} \circ \Phi_{1}$, then

$$
L_{1}: X \rightarrow Z_{1}
$$

is a birational isomorphism (since the stabilizer of $\Phi_{1}(X)$ is trivial).

\section{7}

If $\eta \in N$, then

$$
\begin{aligned}
Z_{1}^{\eta}=L_{1}(X)^{\eta}=L_{1}^{\eta}\left(X^{\eta}\right)=\Psi_{1}^{\eta} \circ \Phi_{1}^{\eta}\left(X^{\eta}\right)=\Psi_{1} \circ \Theta_{1}(\eta)\left(\Phi_{1}(X)\right) & = \\
\Psi_{1} \circ \Phi_{1}(X) & =L_{1}(X)=Z_{1},
\end{aligned}
$$

so $Z_{1}$ can be defined by polynomials with coefficient over $\mathbb{Q}(\sqrt{-7})$.

\section{8}

Next, we proceed to compute explicit equations for $Z_{1}$ and the inverse $L_{1}^{-1}: Z_{1} \rightarrow X$.

The following equalities hold:

$$
\begin{aligned}
x_{1} & =\frac{t_{1}}{3}, \quad x_{2}=\frac{t_{2}}{3}, \quad t_{4}=t_{3} \\
(*) x_{4} & =\frac{\left(t_{3}-x_{3}\right)\left(\frac{t_{1}}{3}-\rho^{4}\right)\left(\frac{t_{1}}{3}-\rho^{5}\right)}{x_{3}+\left(\frac{t_{1}}{3}-\rho^{4}\right)\left(\frac{t_{1}}{3}-\rho^{5}\right)} \\
t_{5} & =\frac{t_{1}^{2}}{3}, \quad t_{6}=\frac{t_{2}^{2}}{3}, \quad t_{8}=t_{7}
\end{aligned}
$$




$$
\begin{gathered}
(* *) x_{4}^{2}=\frac{\left(t_{7}-x_{3}^{2}\right)\left(\frac{t_{1}}{3}-\rho^{4}\right)^{2}\left(\frac{t_{1}}{3}-\rho^{5}\right)^{2}}{x_{3}^{2}+\left(\frac{t_{1}}{3}-\rho^{4}\right)^{2}\left(\frac{t_{1}}{3}-\rho^{5}\right)^{2}} \\
t_{9}=\frac{t_{1}^{3}}{9}, \quad t_{10}=\frac{t_{2}^{3}}{9}, \quad t_{12}=t_{11} \\
(* * *) x_{4}^{3}=\frac{\left(t_{11}-x_{3}^{3}\right)\left(\frac{t_{1}}{3}-\rho^{4}\right)^{3}\left(\frac{t_{1}}{3}-\rho^{5}\right)^{3}}{x_{3}^{3}+\left(\frac{t_{1}}{3}-\rho^{4}\right)^{3}\left(\frac{t_{1}}{3}-\rho^{5}\right)^{3}}
\end{gathered}
$$

Equality $(*)$ permits to obtain $x_{4}$ uniquely in terms of $t_{1}$ and $x_{3}$ and the equation

$$
x_{2}^{2}=\left(x_{1}-1\right)\left(x_{1}-\rho^{3}\right)\left(x_{1}-\rho^{5}\right)\left(x_{1}-\rho^{6}\right)
$$

provides a polynomial equation (relating $t_{1}$ and $\left.t_{2}\right)$ given by $P_{1}\left(t_{1}, t_{2}, t_{3}, t_{7}, t_{11}\right)=0$, where

$$
P_{1}\left(t_{1}, t_{2}, t_{3}, t_{7}, t_{11}\right)
$$

$-81+27\left(1+\left(\rho+\rho^{2}+\rho^{4}\right)\right) t_{1}+9 t_{1}^{2}-3\left(\rho+\rho^{2}+\rho^{4}\right) t_{1}^{3}-t_{1}^{4}+9 t_{2}^{2} \in \mathbb{Q}(\sqrt{-7})\left[t_{1}, t_{2}, t_{3}, t_{7}, t_{11}\right]$.

Equation

$$
x_{3}^{2}=\left(x_{1}-\rho^{2}\right)\left(x_{1}-\rho^{4}\right)\left(x_{1}-\rho^{5}\right)\left(x_{1}-\rho^{6}\right)
$$

permits to obtain the new equation

$$
\text { (1) } x_{3}^{2}=\left(t_{1}-3 \rho^{2}\right)\left(t_{1}-3 \rho^{4}\right)\left(t_{1}-3 \rho^{5}\right)\left(t_{1}-3 \rho^{6}\right) / 81 \text {, }
$$

and the equation

$$
x_{4}^{2}=\left(x_{1}-\rho\right)\left(x_{1}-\rho^{3}\right)\left(x_{1}-\rho^{4}\right)\left(x_{1}-\rho^{5}\right)
$$

provides the equation

$$
\text { (2) } x_{4}^{2}=\left(t_{1}-3 \rho\right)\left(t_{1}-3 \rho^{3}\right)\left(t_{1}-3 \rho^{4}\right)\left(t_{1}-3 \rho^{5}\right) / 81 .
$$

In this way, by replacing the above values for $x_{3}^{2}$ and $x_{4}^{2}$ (obtained in (1) and (2)) in the equality $(* *)$, we obtain the polynomial equation $P_{2}\left(t_{1}, t_{2}, t_{3}, t_{7}, t_{11}\right)=0$, where

$$
P_{2}\left(t_{1}, t_{2}, t_{3}, t_{7}, t_{11}\right)
$$

$$
\begin{array}{r}
27+27\left(\rho+\rho^{2}+\rho^{4}\right)-18 t_{1}-3\left(1+\left(\rho+\rho^{2}+\rho^{4}\right)\right) t_{1}^{2}-2 t_{1}^{3}-t_{1}^{4}+27 t_{7} \in \\
\mathbb{Q}(\sqrt{-7})\left[t_{1}, t_{2}, t_{3}, t_{7}, t_{11}\right] .
\end{array}
$$

Now, if we replace, in equality $(* * *), x_{3}^{3}$ by $x_{3}\left(x_{1}-\rho^{2}\right)\left(x_{1}-\rho^{4}\right)\left(x_{1}-\rho^{5}\right)\left(x_{1}-\rho^{6}\right) / 81$ and $x_{4}^{3}$ by $x_{4}\left(t_{1}-3 \rho\right)\left(t_{1}-3 \rho^{3}\right)\left(t_{1}-3 \rho^{4}\right)\left(t_{1}-3 \rho^{5}\right) / 81$, where $x_{4}$ is given in $(*)$, then we obtain a polynomial which is of degree one in the variable $x_{3}$.

$$
\begin{gathered}
x_{3}=\left(-9 \rho^{2}\left(-162 t_{1}-18 t_{1}^{3}+4 t_{1}^{5}-243\left(1+t_{11}\right)+t_{1}^{2}\left(27-54 t_{3}\right)+6 t_{1}^{4} t_{3}\right)+3(729+\right. \\
\left.18 t_{1}^{4}-6 t_{1}^{5}-27 t_{1}^{3}\left(-6+t_{3}\right)-t_{1}^{6}\left(-2+t_{3}\right)+243 t_{1}\left(3+t_{3}\right)+81 t_{1}^{2}\left(2+t_{11}+t_{3}\right)\right)+\rho^{3}(2187-
\end{gathered}
$$


$\left.t_{1}^{7}+27 t_{1}^{4}\left(-6+t_{3}\right)+9 t_{1}^{5}\left(-3+t_{3}\right)+486 t_{1}^{2} t_{3}+81 t_{1}^{3}\left(1+t_{3}\right)+729 t_{1}\left(1+2 t_{3}\right)\right)+\rho^{5}(2187+$ $\left.27 t_{1}^{4}+12 t_{1}^{6}+t_{1}^{7}-729 t_{1}\left(-1+t_{11}-t_{3}\right)+729 t_{1}^{2} t_{3}+81 t_{1}^{3}\left(5+t_{3}\right)+9 t_{1}^{5}\left(1+2 t_{3}\right)\right)+$ $\rho\left(2916 t_{1}+3 t_{1}^{6}-t_{1}^{7}-81 t_{1}^{3}\left(-6+t_{3}\right)-2187\left(-2+t_{3}\right)-27 t_{1}^{4}\left(-2+t_{3}\right)+9 t_{1}^{5}\left(2+t_{3}\right)+\right.$ $\left.243 t_{1}^{2}\left(5+2 t_{3}\right)\right)+\rho^{4}\left(2187+t_{1}^{7}-729 t_{1}\left(-3+t_{11}-2 t_{3}\right)-81 t_{1}^{3}\left(-1+t_{3}\right)+27 t_{1}^{4}\left(1+t_{3}\right)+\right.$ $\left.\left.9 t_{1}^{5}\left(-1+2 t_{3}\right)+243 t_{1}^{2}\left(1+3 t_{3}\right)\right)\right) /\left(9\left(t_{1}^{5}-243 t_{11}+27 t_{1}^{2}\left(-1+t_{3}\right)+81 t_{1} t_{3}+9 t_{1}^{3} t_{3}+\right.\right.$ $3 t_{1}^{4} t_{3}+\rho\left(3+t_{1}\right)\left(-81+18 t_{1}^{2}-9 t_{1}^{3}+2 t_{1}^{4}+27 t_{1} t_{3}\right)+27 \rho^{2} t_{1}\left(3+t_{1}^{2}+t_{1}\left(3+t_{3}\right)\right)+$ $\rho^{4} t_{1}\left(243+3 t_{1}^{3}+t_{1}^{4}+9 t_{1}^{2}\left(-1+t_{3}\right)+27 t_{1}\left(3+t_{3}\right)\right)+\rho^{5}\left(-6 t_{1}^{4}+t_{1}^{5}+243\left(1+t_{3}\right)+\right.$ $\left.\left.\left.81 t_{1}\left(2+t_{3}\right)+9 t_{1}^{3}\left(2+t_{3}\right)+27 t_{1}^{2}\left(3+t_{3}\right)\right)+\rho^{3} t_{1}\left(162+36 t_{1}^{2}+6 t_{1}^{3}+2 t_{1}^{4}+27 t_{1}\left(4+t_{3}\right)\right)\right)\right)$

Then, using $(*)$, we obtain

$x_{4}=-\left(\left(3 \rho^{4}-t_{1}\right)\left(3 \rho^{5}-t_{1}\right)\left(-\rho^{3}\left(-2187-729 t_{1}+t_{1}^{7}+243 t_{1}^{2} t_{3}\left(2+t_{3}\right)+9 t_{1}^{5}(3+\right.\right.\right.$ $\left.\left.t_{3}\right)+27 t_{1}^{4}\left(6+t_{3}\right)+81 t_{1}^{3}\left(-1+3 t_{3}\right)\right)+\rho^{4}\left(2187+27 t_{1}^{4}+t_{1}^{7}+9 t_{1}^{5}\left(-1+t_{3}\right)-729 t_{1}(-3+\right.$ $\left.\left.t_{11}+t_{3}\right)-243 t_{1}^{2}\left(-1+t_{3}^{2}\right)-81 t_{1}^{3}\left(-1+t_{3}^{2}\right)\right)+\rho\left(4374+486 t_{1}^{3}+54 t_{1}^{4}+3 t_{1}^{6}-t_{1}^{7}-9 t_{1}^{5}(-2+\right.$ $\left.\left.t_{3}\right)-243 t_{1}^{2}\left(-5+t_{3}^{2}\right)-729 t_{1}\left(-4-t_{3}+t_{3}^{2}\right)\right)-3\left(t_{1}^{6}\left(-2+t_{3}\right)+3 t_{1}^{5}\left(2+t_{3}\right)-729(1+\right.$ $\left.t_{11} t_{3}\right)-81 t_{1}^{2}\left(2+t_{11}+2 t_{3}-t_{3}^{2}\right)+9 t_{1}^{4}\left(-2+t_{3}^{2}\right)+243 t_{1}\left(-3-t_{3}+t_{3}^{2}\right)+27 t_{1}^{3}(-6+$ $\left.\left.t_{3}+t_{3}^{2}\right)\right)-9 \rho^{2}\left(4 t_{1}^{5}-243\left(1+t_{11}\right)+81 t_{1}\left(-2+t_{3}\right)+6 t_{1}^{4} t_{3}+9 t_{1}^{3}\left(-2+3 t_{3}\right)+27 t_{1}^{2}(1+\right.$ $\left.\left.t_{3}+t_{3}^{2}\right)\right)+\rho^{5}\left(12 t_{1}^{6}+t_{1}^{7}-243 t_{1}^{2} t_{3}^{2}+9 t_{1}^{5}\left(1+t_{3}\right)+27 t_{1}^{4}\left(1+2 t_{3}\right)-81 t_{1}^{3}\left(-5+t_{3}+\right.\right.$ $\left.\left.\left.\left.t_{3}^{2}\right)-2187\left(-1+t_{3}+t_{3}^{2}\right)-729 t_{1}\left(-1+t_{11}+t_{3}+t_{3}^{2}\right)\right)\right)\right) /\left(9\left(567 t_{1}^{3}+6 t_{1}^{6}+t_{1}^{7}+\rho(-3+\right.\right.$ $\left.t_{1}\right)\left(-54 t_{1}^{3}+t_{1}^{6}+9 t_{1}^{4}\left(-4+t_{3}\right)+729\left(-2+t_{3}\right)+243 t_{1}\left(-2+t_{3}\right)-81 t_{1}^{2}\left(-2+t_{3}\right)\right)+$ $27 t_{1}^{4}\left(-7+t_{3}\right)+9 t_{1}^{5}\left(-5+t_{3}\right)+2187\left(2+t_{3}\right)+243 t_{1}^{2}\left(-1+2 t_{3}\right)+729 t_{1}\left(1+2 t_{3}\right)+$ $\rho^{5}\left(2187+216 t_{1}^{4}+3 t_{1}^{6}+2 t_{1}^{7}+729 t_{1} t_{3}+729 t_{1}^{2}\left(1+t_{3}\right)+18 t_{1}^{5}\left(2+t_{3}\right)+81 t_{1}^{3}\left(16+t_{3}\right)\right)+$ $\rho^{3} t_{1}\left(9 t_{1}^{5}+t_{1}^{6}+27 t_{1}^{3}\left(-4+t_{3}\right)+9 t_{1}^{4}\left(3+t_{3}\right)+81 t_{1}^{2}\left(5+t_{3}\right)+729\left(-5+2 t_{3}\right)+243 t_{1}(-3+\right.$ $\left.\left.2 t_{3}\right)\right)+\rho^{4}\left(2187+6 t_{1}^{6}+2 t_{1}^{7}-81 t_{1}^{3}\left(-14+t_{3}\right)+18 t_{1}^{5}\left(-2+t_{3}\right)+1458 t_{1} t_{3}+27 t_{1}^{4}(5+\right.$ $\left.\left.\left.\left.t_{3}\right)+243 t_{1}^{2}\left(1+3 t_{3}\right)\right)-9 \rho^{2}\left(-243+243 t_{1}-27 t_{1}^{3}+t_{1}^{5}-54 t_{1}^{2}\left(-5+t_{3}\right)+t_{1}^{4}\left(-9+6 t_{3}\right)\right)\right)\right)$.

Now, using such values for $x_{3}$ and $x_{4}$, and replacing them in (1) and (2) above, we obtain another two polynomials identities $P_{3}\left(t_{1}, t_{3}, t_{7}, t_{11}\right)=0$ and $P_{4}\left(t_{1}, t_{3}, t_{7}, t_{11}\right)=0$, where these two new polynomials are defined over $\mathbb{Q}(\rho)$ (see [9] for these long polynomials). In this way, we have obtained the following equations over $\mathbb{Q}(\rho)$ for $Z_{1}$ :

$$
Z_{1}=\left\{\begin{array}{c}
t_{4}=t_{3}, 3 t_{5}=t_{1}^{2}, 3 t_{6}=t_{2}^{2}, t_{8}=t_{9} \\
9 t_{9}=t_{1}^{3}, 9 t_{10}=t_{2}^{3}, t_{12}=t_{11} \\
P_{1}\left(t_{1}, t_{2}, t_{3}, t_{7}, t_{11}\right)=0 \\
P_{2}\left(t_{1}, t_{2}, t_{3}, t_{7}, t_{11}\right)=0 \\
P_{3}\left(t_{1}, t_{2}, t_{3}, t_{7}, t_{11}\right)=0 \\
P_{4}\left(t_{1}, t_{2}, t_{3}, t_{7}, t_{11}\right)=0
\end{array}\right\} \subset \mathbb{C}^{12}
$$

Notice that, by the above computations, we have explicitly the inverse of $L_{1}$ given as

$$
\begin{gathered}
L_{1}^{-1}: Z_{1} \rightarrow X \\
\left(t_{1}, \ldots, t_{12}\right) \mapsto\left(x_{1}, x_{2}, x_{3}, x_{4}\right),
\end{gathered}
$$

where $x_{1}, x_{2}, x_{3}$ and $x_{4}$ are in terms of $t_{1}, t_{2}, t_{3}, t_{7}$ and $t_{11}$.

As the variables $t_{1}, \ldots, t_{12}$ are uniquely determined only by the variables $t_{1}, t_{2}, t_{3}, t_{7}$ and $t_{11}$, if we consider the projection

$$
\begin{aligned}
& \pi: \mathbb{C}^{12} \rightarrow \mathbb{C}^{5} \\
&\left(t_{1}, \ldots, t_{12}\right) \mapsto\left(t_{1}, t_{2}, t_{3}, t_{7}, t_{11}\right),
\end{aligned}
$$


then

$$
\begin{gathered}
L=\pi \circ L_{1}: X \rightarrow Z \\
L_{1}^{*}\left(x, y_{1}, y_{2}, y_{4}\right) \\
\| \\
\left(3 x, 3 y_{1}, y_{2}+y_{4}+\frac{y_{2} y_{4}}{\left(x-\rho^{4}\right)\left(x-\rho^{5}\right)}, y_{2}^{2}+y_{4}^{2}+\frac{y_{2}^{2} y_{4}^{2}}{\left(x-\rho^{4}\right)^{2}\left(x-\rho^{5}\right)^{2}}, y_{2}^{3}+y_{4}^{3}+\frac{y_{2}^{3} y_{4}^{3}}{\left(x-\rho^{4}\right)^{3}\left(x-\rho^{5}\right)^{3}}\right)
\end{gathered}
$$

is a birational isomorphism, where

$$
Z=\left\{\begin{array}{l}
P_{1}\left(t_{1}, t_{2}, t_{3}, t_{7}, t_{11}\right)=0 \\
P_{2}\left(t_{1}, t_{2}, t_{3}, t_{7}, t_{11}\right)=0 \\
P_{3}\left(t_{1}, t_{2}, t_{3}, t_{7}, t_{11}\right)=0 \\
P_{4}\left(t_{1}, t_{2}, t_{3}, t_{7}, t_{11}\right)=0
\end{array}\right\} \subset \mathbb{C}^{5}
$$

The inverse $L^{-1}: Z \rightarrow X$ is given as

$$
L^{-1}\left(t_{1}, t_{2}, t_{3}, t_{7}, t_{11}\right)=\left(x_{1}, x_{2}, x_{3}, x_{4}\right)
$$

We have obtained equations for $Z$ over $\mathbb{Q}(\rho)$. But, as $Z_{1}^{\eta}=Z_{1}$, for every $\eta \in N$, and $\pi$ is defined over $\mathbb{Q}$, we may see that $Z^{\eta}=Z$, for every $\eta \in N$, that is, $Z$ can be defined by polynomials over $\mathbb{Q}(\sqrt{-7})$. To obtain such equations over $\mathbb{Q}(\sqrt{-7})$, we replace each polynomial $P_{j}(j=3,4)$ by the new polynomials (with coefficients in $\mathbb{Q}(\sqrt{-7})$ )

$$
Q_{j, 1}=\operatorname{Tr}\left(P_{j}\right), Q_{j, 2}=\operatorname{Tr}\left(\rho P_{j}\right), Q_{j, 3}=\operatorname{Tr}\left(\rho^{2} P_{j}\right)
$$

that is

$$
Z=\left\{\begin{array}{c}
P_{1}\left(t_{1}, t_{2}, t_{3}, t_{7}, t_{11}\right)=0 \\
P_{2}\left(t_{1}, t_{2}, t_{3}, t_{7}, t_{11}\right)=0 \\
P_{3}\left(t_{1}, t_{2}, t_{3}, t_{7}, t_{11}\right)+P_{3}\left(t_{1}, t_{2}, t_{3}, t_{7}, t_{11}\right)^{\tau}+P_{3}\left(t_{1}, t_{2}, t_{3}, t_{7}, t_{11}\right)^{\tau^{2}}=0 \\
\rho P_{3}\left(t_{1}, t_{2}, t_{3}, t_{7}, t_{11}\right)+\rho^{2} P_{3}\left(t_{1}, t_{2}, t_{3}, t_{7}, t_{11}\right)^{\tau}+\rho^{4} P_{3}\left(t_{1}, t_{2}, t_{3}, t_{7}, t_{11}\right)^{\tau^{2}}=0 \\
\rho^{2} P_{3}\left(t_{1}, t_{2}, t_{3}, t_{7}, t_{11}\right)+\rho^{4} P_{3}\left(t_{1}, t_{2}, t_{3}, t_{7}, t_{11}\right)^{\tau}+\rho P_{3}\left(t_{1}, t_{2}, t_{3}, t_{7}, t_{11}\right)^{\tau^{2}}=0 \\
P_{4}\left(t_{1}, t_{2}, t_{3}, t_{7}, t_{11}\right)+P_{4}\left(t_{1}, t_{2}, t_{3}, t_{7}, t_{11}\right)^{\tau}+P_{4}\left(t_{1}, t_{2}, t_{3}, t_{7}, t_{11}\right)^{\tau^{2}}=0 \\
\rho P_{4}\left(t_{1}, t_{2}, t_{3}, t_{7}, t_{11}\right)+\rho^{2} P_{4}\left(t_{1}, t_{2}, t_{3}, t_{7}, t_{11}\right)^{\tau}+\rho^{4} P_{4}\left(t_{1}, t_{2}, t_{3}, t_{7}, t_{11}\right)^{\tau^{2}}=0 \\
\rho^{2} P_{4}\left(t_{1}, t_{2}, t_{3}, t_{7}, t_{11}\right)+\rho^{4} P_{4}\left(t_{1}, t_{2}, t_{3}, t_{7}, t_{11}\right)^{\tau}+\rho P_{4}\left(t_{1}, t_{2}, t_{3}, t_{7}, t_{11}\right)^{\tau^{2}}=0
\end{array}\right\} \subset \mathbb{C}^{5}
$$

We have obtained an explicit model $Z$ for the Fricke-Macbeath curve over $\mathbb{Q}(\sqrt{-7})$ together explicit birational isomorphisms $L: X \rightarrow Z$ and $L^{-1}: Z \rightarrow X$.

\section{9}

Finally, notice that the regular dessin d'enfant $(X, \beta)$, given before, is isomorphic to that provided by the pair $\left(Z, \beta^{*}\right)$, where $\beta^{*}\left(t_{1}, t_{2}, t_{3}, t_{7}, t_{11}\right)=\beta \circ L^{-1}\left(t_{1}, t_{2}, t_{3}, t_{7}, t_{11}\right)=$ $\left(t_{1} / 3\right)^{7}$; that is, the dessin d'enfant is defined over $\mathbb{Q}(\sqrt{-7})$. 


\section{An explicit isomorphism $L: X \rightarrow W$ where $W$ is defined over $\mathbb{Q}$}

Next we explain how to construct an explicit birational isomorphism $\widehat{L}: X \rightarrow W$, where $W$ is known to be defined over $\mathbb{Q}$.

Let us consider the explicit model $Z \subset \mathbb{C}^{5}$ over $\mathbb{Q}(\sqrt{-7})$ constructed above. Let $M=\operatorname{Gal}(\mathbb{Q}(\sqrt{-7}) / \mathbb{Q})=\langle\eta\rangle \cong \mathbb{Z}_{2}$, where $\eta$ is the complex conjugation. As already noticed, since $X$ admits the anticonformal involution $J$ (defined previously), the curve $Z$ admits the anticonformal involution $T=L \circ J \circ L^{-1}$. It is not difficult to see that by setting $g_{e}=I$ and $g_{\eta}=S \circ T$, where $S\left(t_{1}, t_{2}, t_{3}, t_{7}, t_{11}\right)=\left(\overline{t_{1}}, \overline{t_{2}}, \overline{t_{3}}, \overline{t_{7}}, \overline{t_{11}}\right)$, we obtain a Weil datum for the Galois extension $\mathbb{Q}(\sqrt{-7}) / \mathbb{Q}$. Now, identically as done above, we consider the rational map

$$
\begin{gathered}
\Phi_{2}: Z \rightarrow \mathbb{C}^{10} \\
\left(t_{1}, t_{2}, t_{3}, t_{7}, t_{11}\right) \mapsto\left(t_{1}, t_{2}, t_{3}, t_{7}, t_{11}, s_{1}, s_{2}, s_{3}, s_{7}, s_{11}\right)
\end{gathered}
$$

where $g_{\eta}\left(t_{1}, t_{2}, t_{3}, t_{7}, t_{11}\right)=\left(s_{1}, s_{2}, s_{3}, s_{7}, s_{11}\right)$. We may see that $\Phi_{2}$ induces a birational isomorphism between $Z$ and $\Phi_{2}(Z)$. In this case,

$$
\Phi_{2}(Z)=\left\{\begin{array}{c}
Q_{1,1}\left(t_{1}, t_{2}, t_{3}, t_{7}, t_{11}\right)=\cdots=Q_{4,3}\left(t_{1}, t_{2}, t_{3}, t_{7}, t_{11}\right)=0 \\
g_{\eta}\left(t_{1}, t_{2}, t_{3}, t_{7}, t_{11}\right)=\left(s_{1}, s_{2}, s_{3}, s_{7}, s_{11}\right)
\end{array}\right\} \subset \mathbb{C}^{10} .
$$

The Galois group $M$ induces the permutation action $\Theta_{2}(M)$ defined as

$$
\Theta_{2}(\eta)\left(t_{1}, t_{2}, t_{3}, t_{7}, t_{11}, s_{1}, s_{2}, s_{3}, s_{7}, s_{11}\right)=\left(s_{1}, s_{2}, s_{3}, s_{7}, s_{11}, t_{1}, t_{2}, t_{3}, t_{7}, t_{11}\right)
$$

A set of generators for the invariant polynomials (with respect to the previous permutation action) is given by

$$
\begin{gathered}
q_{1}=t_{1}+s_{1}, q_{2}=t_{2}+s_{2}, q_{3}=t_{3}+s_{3}, \\
q_{4}=t_{7}+s_{7}, q_{5}=t_{11}+s_{11}, q_{6}=t_{1}^{2}+s_{1}^{2}, \\
q_{7}=t_{2}^{2}+s_{2}^{2}, q_{8}=t_{3}^{2}+s_{3}^{2}, q_{9}=t_{7}^{2}+s_{7}^{2}, \\
q_{10}=t_{11}^{2}+s_{11}^{2}
\end{gathered}
$$

Then the rational map

$$
\begin{aligned}
\Psi_{2}: \mathbb{C}^{10} & \rightarrow \mathbb{C}^{10} \\
\left(t_{1}, t_{2}, t_{3}, t_{7}, t_{11}, s_{1}, s_{2}, s_{3}, s_{7}, s_{11}\right) & \mapsto\left(q_{1}, q_{2}, q_{3}, q_{4}, q_{5}, q_{6}, q_{7}, q_{8}, q_{9}, q_{10}\right)
\end{aligned}
$$

satisfies the following properties:

$$
\left\{\begin{array}{l}
\Psi_{2}^{\eta}=\Psi_{2} \\
\Psi_{2} \circ \Theta_{2}(\eta)=\Psi_{2}
\end{array}\right.
$$

There are two possibilities:

1. $\Phi_{2}(Z)=\Theta_{2}(\eta)\left(\Phi_{2}(Z)\right)$; in which case $Z^{\eta}=Z$ and $Z$ will be already defined over $\mathbb{Q}$ (which seems not to be the case); and

2. the stabilizer of $\Phi_{2}(Z)$ under $\Theta_{2}(M)$ is trivial. 
Under the assumption (2) above, we have that $\Psi_{2}: \Phi_{2}(Z) \rightarrow W=\Psi_{2}\left(\Phi_{2}(Z)\right)$ is a biregular isomorphism and that, as before, $W$ is defined over $\mathbb{Q}$. That is, the map $L_{1}=\Psi_{2} \circ \Phi_{2}: Z \rightarrow W$ is an explicit biregular isomorphism and $W$ is defined over $\mathbb{Q}$. In this way, $\widehat{L}=L_{1} \circ L: X \rightarrow W$ is an explicit birational isomorphism as desired.

As $R_{2}$ and $Z$ are explicitly given, one may compute explicit equations for $W$ over $\mathbb{Q}(\sqrt{-7})$, say by the polynomials $A_{1}, \ldots, A_{m} \in \mathbb{Q}(\sqrt{-7})\left[q_{1}, \ldots, q_{10}\right]$ (this may be done with MAGMA [3] or by hands, but computations are heavy and very long). To obtain equations over $\mathbb{Q}$ we replace each $A_{j}$ (which is not already defined over $\mathbb{Q}$ ) by the traces $A_{j}+A_{j}^{\eta}$ and $i A_{j}-i A_{j}^{\eta}$.

\section{A remark on the elliptic curves in the model $X$}

\subsection{A connection to homology covers}

Let us set $\lambda_{1}=1, \lambda_{2}=\rho, \lambda_{3}=\rho^{2}, \lambda_{4}=\rho^{3}, \lambda_{5}=\rho^{4}, \lambda_{6}=\rho^{5}$ and $\lambda_{7}=\rho^{6}$, where $\rho=$ $e^{2 \pi i / 7}$. If $S$ is the Fricke-Macbeath curve, then there is a regular branched cover $Q: S \rightarrow \widehat{\mathbb{C}}$ having deck group $G \cong \mathbb{Z}_{2}^{3}$ and whose branch locus is the set $\left\{\lambda_{1}, \lambda_{2}, \lambda_{3}, \lambda_{4}, \lambda_{5}, \lambda_{6}, \lambda_{7}\right\}$.

Let us consider a Fuchsian group

$$
\Gamma=\left\langle\alpha_{1}, \ldots, \alpha_{7}: \alpha_{1}^{2}=\cdots=\alpha_{7}^{2}=\alpha_{1} \alpha_{2} \cdots \alpha_{7}=1\right\rangle
$$

acting on the hyperbolic plane $\mathbb{H}^{2}$ uniformizing the orbifold $S / G$.

If $\Gamma^{\prime}$ denotes the derived subgroup of $\Gamma$, then $\Gamma^{\prime}$ acts freely and $\widehat{S}=\mathbb{H}^{2} / \Gamma^{\prime}$ is a closed Riemann surface. Let $H=\Gamma / \Gamma^{\prime} \cong \mathbb{Z}_{2}^{6}$; a group of conformal automorphisms of $\widehat{S}$. Then there exists a set of generators of $H$, say $a_{1}, \ldots, a_{6}$, so that the only elements of $H$ acting with fixed points are these and $a_{7}=a_{1} a_{2} a_{3} a_{4} a_{5} a_{6}$. In [4, 5] it was noted that $\widehat{S}$ corresponds to the generalized Fermat curve of type $(2,6)$ (also called the homology cover of $S / H)$

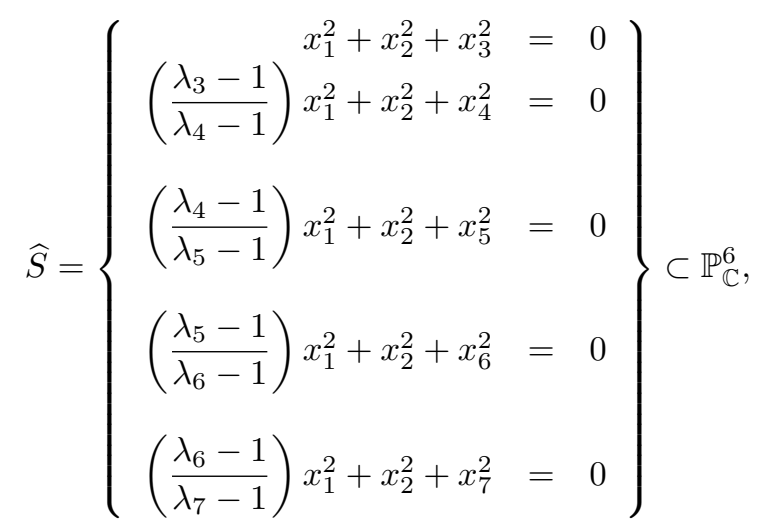

that $a_{j}$ is just multiplication by -1 at the coordinate $x_{j}$ and that the regular branched cover $P: \widehat{S} \rightarrow \widehat{\mathbb{C}}$ given by

$$
P\left(\left[x_{1}: x_{2}: x_{3}: x_{4}: x_{5}: x_{6}: x_{7}\right]\right)=\frac{x_{2}^{2}+x_{1}^{2}}{x_{2}^{2}+\lambda_{7} x_{1}^{2}}=z
$$

has $H$ has its deck group and branch locus given by the set of the $7 t h$-roots of unity $\left\{\lambda_{1}, \ldots, \lambda_{7}\right\}$. 
By classical covering theory, there should be a subgroup $K<H, K \cong \mathbb{Z}_{2}^{3}$, acting freely on $\widehat{S}$ so that there is an isomorphism $\phi: S \rightarrow \widehat{S} / K$ with $\phi G \phi^{-1}=H / K$.

Let us also observe that the rotation $R(z)=\rho z$ lifts under $P$ to an automorphism $T$ of $\widehat{S}$ of order 7 of the form

$$
T\left(\left[x_{1}: \cdots: x_{7}\right]\right)=\left[c_{1} x_{7}: c_{2} x_{1}: c_{3} x_{2}: c_{4} x_{3}: c_{5} x_{4}: c_{6} x_{5}: c_{7} x_{6}\right]
$$

for suitable comples numbers $c_{j}$. One has that $T a_{j} T^{-1}=a_{j+1}$, for $j=1, \ldots, 6$ and $T a_{7} T^{-1}=a_{1}$. The subgroup $K$ above must satisfy that $T K T^{-1}=K$ as $R$ also lifts to the Fricke-Macbeath curve (as noticed in the Introduction).

\subsection{About the elliptic curves in the Fricke-Macbeath curve}

The subgroup

$$
K^{*}=\left\langle a_{1} a_{3} a_{7}, a_{2} a_{3} a_{5}, a_{1} a_{2} a_{4}\right\rangle \cong \mathbb{Z}_{2}^{3}
$$

acts freely on $\widehat{S}$ and it is normalized by $T$. In particular, $S^{*}=\widehat{S} / K^{*}$ is a closed Riemann surface of genus 7 admitting the group $L=H / K^{*}=\left\{e, a_{1}^{*}, \ldots, a_{7}^{*}\right\} \cong \mathbb{Z}_{2}^{3}$ (where $a_{j}^{*}$ is the involution induced by $a_{j}$ ) as a group of automorphisms and it also has an automorphism $T^{*}$ of order 7 (induced by $T$ ) permuting cyclically the involutions $a_{j}^{*}$. As $S^{*} /\left\langle L, T^{*}\right\rangle=$ $\widehat{S} /\langle H, T\rangle$ has signature $(0 ; 2,7,7)$, we may see that $S=S^{*}$ and $K=K^{*}$.

We may see that $L=\left\langle a_{1}^{*}, a_{2}^{*}, a_{3}^{*}\right\rangle$ and $a_{4}^{*}=a_{1}^{*} a_{2}^{*}, a_{5}^{*}=a_{2}^{*} a_{3}^{*}, a_{6}^{*}=a_{1}^{*} a_{2}^{*} a_{3}^{*}$ and $a_{7}^{*}=a_{1}^{*} a_{3}^{*}$. In this way, we may see that every involution of $H / K$ is induced by one of the involutions (and only one) with fixed points; so every involution in $L$ acts with 4 fixed points on $S$.

Let $a_{i}^{*}, a_{j}^{*} \in H / K$ be any two different involutions, so $\left\langle a_{i}^{*}, a_{j}^{*}\right\rangle \cong \mathbb{Z}_{2}^{2}$. Then, by the Riemann-Hurwitz formula, the quotient surface $S /\left\langle a_{i}^{*}, a_{j}^{*}\right\rangle$ is a closed Riemann surface of genus 1 with six cone points of order 2 . These six cone points are projected onto three of the cone points of $S / H$. These points are $\lambda_{i}, \lambda_{j}$ and $\lambda_{r}$, where $a_{r}^{*}=a_{i}^{*} a_{j}^{*}$. In this way, the corresponding genus one surface is given by the elliptic curve

$$
y^{2}=\prod_{k \notin\{i, j, r\}}\left(x-\lambda_{k}\right)
$$

So, for instance, if we consider $i=2$ and $j=3$, then $r=5$ and the elliptic curve is

$$
y_{1}^{2}=(x-1)\left(x-\rho^{3}\right)\left(x-\rho^{5}\right)\left(x-\rho^{6}\right) .
$$

If $i=1$ and $j=2$, then $r=4$ and the elliptic curve is

$$
y_{2}^{2}=\left(x-\rho^{2}\right)\left(x-\rho^{4}\right)\left(x-\rho^{5}\right)\left(x-\rho^{6}\right) .
$$

If $i=1$ and $j=3$, then $r=7$ and the elliptic curve is

$$
y_{4}^{2}=(x-\rho)\left(x-\rho^{3}\right)\left(x-\rho^{4}\right)\left(x-\rho^{5}\right) .
$$

We have obtained the three elliptic curves appearing in the Fricke-Macbeath equation (2.1). 


\subsection{Another model for the Fricke-Macbeath curve}

The above description of the Fricke-Macbeath curve in terms of the homology cover $\widehat{S}$ permits to obtain an explicit model. Let us consider now an affine model of $\widehat{S}$, say by taking $x_{7}=1$, which we denote by $\widehat{S}^{0}$. In this case the involution $a_{7}$ is multiplication of all coordinates by -1 . A set of generators for the algebra of invariant polynomials in $\mathbb{C}\left[x_{1}, x_{2}, x_{3}, x_{4}, x_{5}, x_{6}\right]$ under the natural linear action induced by $K$ is

$$
\begin{gathered}
t_{1}=x_{1}^{2}, t_{2}=x_{2}^{2}, t_{3}=x_{3}^{2}, t_{4}=x_{4}^{2}, t_{5}=x_{5}^{2}, t_{6}=x_{6}^{2}, t_{7}=x_{1} x_{2} x_{5}, t_{8}=x_{1} x_{2}, x_{3} x_{6} \\
t_{9}=x_{1} x_{4} x_{6}, t_{10}=x_{1} x_{3} x_{4} x_{5}, t_{11}=x_{2} x_{4} x_{5} x_{6}, t_{12}=x_{2} x_{3} x_{4}, t_{13}=x_{3} x_{5} x_{6} .
\end{gathered}
$$

If we set

$$
\begin{gathered}
F: \widehat{S}^{0} \rightarrow \mathbb{C}^{13} \\
F\left(x_{1}, x_{2}, x_{3}, x_{4}, x_{5}, x_{6}\right)=\left(t_{1}, t_{2}, t_{3}, t_{4}, t_{5}, t_{6}, t_{7}, t_{8}, t_{9}, t_{10}, t_{11}, t_{12}, t_{13}\right),
\end{gathered}
$$

then $F\left(\widehat{S}^{0}\right)$ will provide a model for the Fricke-Macbeath (affine) curve $S$. Equations for such an affine model of $S$ are

$$
\left\{\begin{array}{c}
t_{1}+t_{2}+t_{3}=0 \\
\left(\frac{\lambda_{3}-1}{\lambda_{4}-1}\right) t_{1}+t_{2}+t_{4}=0 \\
\left(\frac{\lambda_{4}-1}{\lambda_{5}-1}\right) t_{1}+t_{2}+t_{5}=0 \\
\left(\frac{\lambda_{5}-1}{\lambda_{6}-1}\right) t_{1}+t_{2}+t_{6}=0 \\
\left(\frac{\lambda_{6}-1}{\lambda_{7}-1}\right) t_{1}+t_{2}+1=0 \\
t_{6} t_{10}=t_{9} t_{13}, t_{6} t_{7} t_{12}=t_{8} t_{11}, t_{5} t_{9} t_{12}=t_{10} t_{11} \\
t_{5} t_{8}=t_{7} t_{13}, t_{5} t_{6} t_{12}=t_{11} t_{13}, t_{4} t_{8}=t_{9} t_{12} \\
t_{4} t_{7} t_{13}=t_{10} t_{11}, t_{4} t_{6} t_{7}=t_{9} t_{11}, t_{3} t_{11}=t_{12} t_{13} \\
t_{3} t_{6} t_{7}=t_{8} t_{13}, t_{3} t_{5} t_{9}=t_{10} t_{13}, t_{3} t_{5} t_{6}=t_{13}^{2} \\
t_{3} t_{4} t_{7}=t_{10} t_{12}, t_{2} t_{10}=t_{7} t_{12}, t_{2} t_{9} t_{13}=t_{8} t_{11} \\
t_{2} t_{5} t_{9}=t_{7} t_{11}, t_{2} t_{4} t_{13}=t_{11} t_{12}, t_{2} t_{4} t_{5} t_{6}=t_{11}^{2} \\
t_{2} t_{3} t_{9}=t_{8} t_{12}, t_{2} t_{3} t_{4}=t_{12}^{2}, t_{1} t_{12} t_{13}=t_{8} t_{10} \\
t_{1} t_{11}=t_{7} t_{9}, t_{1} t_{6} t_{12}=t_{8} t_{9}, t_{1} t_{5} t_{12}=t_{7} t_{10} \\
t_{1} t_{4} t_{13}=t_{9} t_{10}, t_{1} t_{4} t_{6}=t_{9}^{2}, t_{1} t_{3} t_{4} t_{5}=t_{10}^{2} \\
t_{1} t_{2} t_{13}=t_{7} t_{8}, t_{1} t_{2} t_{5}=t_{7}^{2}, t_{1} t_{2} t_{3} t_{6}=t_{8}^{2}
\end{array}\right\} \subset \mathbb{C}^{13}
$$

Of course, one may see that the variables $t_{2}, t_{3}, t_{4}, t_{5}$ and $t_{6}$ are uniquely determined by the variable $t_{1}$. Other variables can also be determined in order to get a lower dimensional model.

\section{Acknowledgments}

The author is grateful to the referee whose suggestions, comments and corrections done to the preliminary versions helped to improve the presentation of the paper. I also want to thanks J. Wolfart for many early discussions about the results obtained in here. 


\section{References}

[1] G. V. Bely̌̌, On Galois extensions of a maximal cyclotomic field, Izv. Akad. Nauk SSSR Ser. Mat. 43 (1979), 267-276, 479.

[2] K. Berry and M. Tretkoff, The period matrix of Macbeath's curve of genus seven, Curves, Jacobians, and abelian varieties (Amherst, MA, 1990), 31-40, Contemp. Math., 136, Amer. Math. Soc., Providence, RI, 1992.

[3] W. Bosma, J. Cannon and C. Playoust, The Magma algebra system, I. The user language, Computational algebra and number theory (London, 1993), J. Symbolic Comput. 24 (1997), 235-265, http://dx.doi.org/10.1006/jsco.1996.0125

[4] A. Carocca, V. González-Aguilera, R. A. Hidalgo and R. E. Rodríguez, Generalized Humbert curves, Israel J. Math. 164 (2008), 165-192.

[5] G. González-Diez, R.A. Hidalgo and M. Leyton, Generalized Fermat curves, Journal of Algebra 321 (2009), 1643-1660.

[6] T. Ekedahl and J.-P. Serre, Exemples de courbes algébriques à jacobienne complètement décomposable, C. R. Acad. Sci. Pari Sér. I Math. 317 (1993), 509-513.

[7] M. Hendriks, Platonic maps of low genus, Ph. D. Thesis, Technische Universiteit Eindhoven, 2013

[8] R. A. Hidalgo and S. Reyes-Carocca, Towards a constructive proof of Weil's descent theorem, http: //arxiv.org/abs/1203.6294

[9] R. A. Hidalgo. A computational note about Fricke-Macbeath's curve. http: / / arxiv • org/ abs/1203.6314

[10] L. D. James and G. A. Jones, Regular orientable imbeddings of complete graphs, J. Combin. Theory Ser. B 39 (1985), 353-367.

[11] G. A. Jones and D. Singerman, Theory of maps on orientable surfaces, Proc. London Math. Soc. 37 (1978), 273-307.

[12] G. A. Jones, M. Streit and J. Wolfart, Wilson's map operations on regular dessins and cyclotomic fields of definition, Proc. Lond. Math. Soc. 100 (2010), 510-532.

[13] S. K. Lando and A. Zvonkin, Graphs on surfaces and their applications, with an appendix by Don B. Zagier, Encyclopaedia of Mathematical Sciences 141, Low-Dimensional Topology, II. Springer-Verlag, Berlin, 2004.

[14] A. Macbeath, On a curve of genus 7, Proc. London Math. Soc. 15 (1965), 527-542.

[15] D. Singerman. Automorphisms of maps, permutation groups and Riemann surfaces. Bull. London Math. Soc. 8 (1976), 65-68.

[16] A. Weil, The field of definition of a variety, Amer. J. Math. 78 (1956), 509-524.

[17] A. Wiman, "Ueber die hyperelliptischen Curven und diejenigen vom Geschlechte $p=3$, welche eindeutigen Transformationen in sich zulassen" and "Ueber die algebraischen Curven von den Geschlechtern $p=4,5$ und 6 welche eindeutigen Transformationen in sich besitzen", Bihang Till Kongl. Svenska Vetenskaps-Akademiens Hadlingar, Stockholm, 1895-96.

[18] J. Wolfart (joint work with G. Jones and M. Streit), Wilson's operations on regular dessins and cyclotomic fields of definition, Talk in Ankara, March 2011.

[19] J. Wolfart, ABC for polynomials, dessins d'enfants and uniformization - a survey. Elementare und analytische Zahlentheorie, 313-345, Schr. Wiss. Ges. Johann Wolfgang Goethe Univ. Frankfurt am Main, 20, Franz Steiner Verlag Stuttgart, Stuttgart, 2006.

[20] Wolfram Research, Inc., Mathematica, Version 8.0, Champaign, IL (2010). 